Nuclear Physics B (Proc. Suppl.) 99A (2013) 1-1

North-Holland

\title{
CLASSICAL DYNAMICS OF POINT PARTICLES IN 2+1 GRAVITY
}

\author{
Andrea Cappelli \\ Theory Division, CERN, Geneva, Switzerland (On leave from INFN, Firenze, Italy)
}

\begin{abstract}
The relation between Einstein gravity and the Chern-Simons gauge theory of the Poincaré group is discussed at the classical level. The general form of the gauge field is described in the presence of point sources, corresponding to non-abelian anyons. They have arbitrary motion apart from particle exchanges, which are governed by the braid group. The gravitational classical interaction appears when a specific gauge fixing is taken, such that the metric is smooth and invertible, with proper asymptotic behaviour. Once the asymptotic motion is fixed, the classical two-body scattering problem is well defined.
\end{abstract}

\section{Introduction}

Three-dimensional gravity is an interesting laboratory for understanding both classical and quantum properties of field theories with reparametrization invariance [1]2]. Simple examples and exact solutions allow a non-verbose approach to their many puzzling issues. Besides, it has physical applications to cosmic strings in four dimensions [3].

Here we shall limit ourselves to introducing matter by $N$ (dynamical) point sources,

$S=-\frac{1}{16 \pi G_{N}} \int d^{3} x \sqrt{g} \mathcal{R}-\sum_{(r)=1}^{N} m_{(r)} \int d s_{(r)}(1)$

In the next section, we recall that this theory can be rewritten as a Chern-Simons gauge theory of the Poincaré group $I S O(2,1)$, by using the first-order (Palatini) formalism [2]. Namely, the long-standing hope of relating gravity to gauge theory becomes a reality. The peculiar aspects of this relation form the body of the following discussion \#1.

\#1 Based on the work done in collaboration with M. Ciafaloni and P.Valtancoli 4. 4 .
At the quantum level, the Chern-Simons approach suggests that $2+1$ gravity is renormalizable, in spite of non-renormalizability by power counting of the Einstein-Hilbert action (11). Actually, the two approaches are related by a nonlinear change of variables, involving a dimensionful scale, thus the two different perturbative expansions correspond to different "phases" of gravity, with unbroken $\left\langle g_{\mu \nu}\right\rangle=0$ and broken $\left\langle g_{\mu \nu}\right\rangle \sim \eta_{\mu \nu}$ reparametrization invariance, respectively.

We would like to show that an insight in this still vague issue comes from the classical problem of determining the motion of point particles under their own field. Actually, this can be tackled and its solution shows some characteristic differences between gauge theory and gravity.

The problem of motion in gravity was first studied by Einstein,Infeld and Hoffmann [6] in four dimensions, and it has never been solved beyond perturbation theory, due to its formidable non-linearity. In three dimensions, some exact many-particle space-times were found by Deser, Jackiw and 't Hooft [1], owing to the following simplifing features: i) there is no on-shell gravi- 
ton, ii) nor Newton static potential, and iii) the space is flat outside sources. Still, exact solutions were missing for two or more moving particles. This is recalled in sect. 3 and fully discussed in the reviews $[7$.

On the other hand, the anyon problem in Chern-Simons theory can be easily solved completely [4]. Amazingly, the motion is almost arbitrary, because the trajectories of particles are gauge degrees of freedom and can be deformed at will. This solution is shown in sect. 4 , in a generic gauge. The unique constraint is that topologically inequivalent trajectories (i.e. particle exchanges) are related by the braid group. This acts on the topological observables, which are the holonomies of the gauge field around each particle 8.9.

In sect. 5, we show that the anyon problem indeed corresponds to gravity by choosing an appropriate gauge-fixing, in which the special properties of the metric field with respect to the gauge field can be met\#2. Namely, the metric should be smooth and invertible, outside particle trajectories, and asymptotically constant. Instead, the simpler anyon solutions give, in general, noninvertible metrics, which, by definition, are not metrics. The (singular) gauge transformation between the two cases was called $\Lambda$-transformation [4].

Therefore, the main difference between gauge theory and gravity lies in the relevance of the gauge choice, a problem complementary or "dual" to the solution of the equations of motion. Actually, it is the hardest one in our approach, because it brings back non-linearities into the problem.

Of course, the complete details of the trajectories (i.e. of the gauge) should not matter

\#2 Somewhat similar statements are also found in the refs. 10 11]. for the quantum theory, which should be locally gauge invariant. However, the asymptotic gaugefixing is somehow unavoidable, because it allows to identify the "asymptotic states" (asymptotic metric), and to define the two-body scattering. The holonomies for open paths going to \pm infinity are similarly well defined, and yield new observables. The time-like open path gives the scattering angle, which we obtain exactly for massless particles.

In the conclusion, some open problems suggested by this classical analysis are discussed.

\section{Gravity in the First-Order Formalism and Chern-Simons Theory}

In the usual second-order formalism $(g, \Gamma[g])$, the metric is the field variable. In the first-order formalism $(e, \omega)$, two field variables are introduced, the basis vectors of the moving frame, or dreibeins $e_{\mu}^{a}$, and the "spin" connection $\omega_{\mu b}^{a}$ for the associated local Lorentz invariance. They are related by the equations

$$
\begin{aligned}
& g_{\mu \nu}=e_{\mu}^{a} \eta_{a b} e^{b}{ }_{\nu} \quad \text { (soldering condition) (2) } \\
& \Gamma_{\mu \nu}^{\lambda}=\left(e^{-1}\right)_{a}^{\lambda}\left(\delta_{b}^{a} \partial_{\mu}+\omega_{\mu b}^{a}\right) e_{\nu}^{b} \quad \text { (metricity) (3) }
\end{aligned}
$$

The first equation states how to connect the Lorentz manifold to space-time, the second one defines the parallel transport which preserves the metric, $D_{\mu}[\Gamma] g_{\alpha \beta}=0$. A third equation involves the particle trajectory $\xi(\tau)$. The space-time momentum

$p_{(r)}^{\mu} \equiv m_{(r)} \dot{\xi}_{(r)}^{\mu}, \quad\left(\dot{\xi} \equiv \frac{d x}{d \tau}\right)$

is related to the Lorentz momentum $P^{a}$ by

$p_{(r)}^{\mu} e_{\mu}^{a}\left(\xi_{(r)}\right)=P_{(r)}^{a},($ particle kinematics $)(5)$ 
Thanks to the three-dimensional identity

$\epsilon^{\mu \nu \rho} \epsilon_{a b c} e_{\mu}^{a} e_{\nu}^{b} e_{\rho}^{c}=|e| \quad(D=3)$

the Einstein action (11) can be rewritten \#3 (hereafter $8 \pi G_{N} \equiv 1$ )

$$
\begin{aligned}
S= & -\frac{1}{2} \int d^{3} x \epsilon^{\mu \nu \rho} \epsilon_{a b c} e_{\rho}^{a}\left(\partial_{[\mu} \omega_{\nu]}+\omega_{[\mu} \omega_{\nu]}\right)^{b c} \\
& -2 \sum_{(r)} \int d \tau\left[\dot{\xi}^{\mu}\left(P_{a} e_{\mu}^{a}-\frac{1}{2} J_{a} \epsilon^{a b c} \omega_{\mu b c}\right)\right]_{(r)} \\
= & \int\left\langle A,\left(\mathrm{~d} A+\frac{2}{3} A^{2}\right)\right\rangle-2 \sum_{(r)} \oint_{(r)} A
\end{aligned}
$$

The explicit Chern-Simons form in the last equation follows by introducing a gauge connection taking values in the Poincaré group $\operatorname{ISO}(2,1)$

$A_{\mu}=e^{a}{ }_{\mu} \mathcal{P}_{a}-\frac{1}{2} \epsilon^{a b c} \omega_{\mu b c} \mathcal{J}_{a}$

whose generators satisfy

$$
\begin{aligned}
& {\left[\mathcal{J}_{a}, \mathcal{J}_{b}\right]=\epsilon_{a b c} \mathcal{J}^{c}, \quad\left[\mathcal{J}_{a}, \mathcal{P}_{b}\right]=\epsilon_{a b c} \mathcal{P}^{c}} \\
& {\left[\mathcal{P}_{a}, \mathcal{P}_{b}\right]=0}
\end{aligned}
$$

Furthermore, the gauge indices are contracted by using the invariant non-degenerate metric

$$
\left\langle\mathcal{J}_{a}, \mathcal{P}_{b}\right\rangle=\eta_{a b},\left\langle\mathcal{P}_{a}, \mathcal{P}_{b}\right\rangle=0,\left\langle\mathcal{J}_{a}, \mathcal{J}_{b}\right\rangle=0 \text { (10) }
$$

Note that in the Chern-Simons theory $e_{\mu}^{a} \rightarrow$ $e^{a}{ }_{\mu} / G_{N}$, thus $\operatorname{dim}(e)=\operatorname{dim}(\omega)=1$, and the dimensionless gauge coupling constant is not quantized because $\pi_{3}(\operatorname{ISO}(2,1))=0$ [2].

The transformation (14) had miraculous results. We started from the highly non-linear theory (1), not renormalizable by power counting $\left(\operatorname{dim} G_{N}=-1\right)$, and we arrived at the polynomial action (7), which is renormalizable by expanding around the classical solution

$e_{\mu}^{a} \sim 0, \quad \omega_{\mu b}^{a} \sim 0$

\#3 We consider here spinless particles, $J_{(r)}^{a}=0$. which is reparametrization invariant.

Let us pause and think what this "phase" of gravity with unbroken symmetry might be ${ }^{\# 4}$.

Let us consider a quantum theory of gravity where the metric is the fundamental field $\hat{g}_{\mu \nu}$. Next, let us ask what is $\left\langle\hat{g}_{\mu \nu}\right\rangle$, the vacuum expectation value, or better said, the semi-classical mean value.

The broken phase $\left\langle\hat{g}_{\mu \nu}\right\rangle \sim \eta_{\mu \nu}$ (at least for large distances) is the one of the classical limit, with geometry and light-cones. This is better described by the Einstein action (11), which however has the problem of non-renormalizable fluctuations $\hat{g}_{\mu \nu}=\eta_{\mu \nu}+\hat{h}_{\mu \nu}, \hat{h}_{\mu \nu} \ll 1$, with reparametrization invariance broken to Poincaré global symmetry.

The unbroken phase is instead characterized by $\left\langle\hat{g}_{\mu \nu}\right\rangle \equiv g_{\mu \nu}=0$. This equation is however not completely right, because $g_{\mu \nu}^{-1}$ does not exist, thus $g_{\mu \nu}$ cannot be called a metric and geometry is not well-defined. Thus the metric cannot be a fundamental field in this phase, or the other way around, this phase cannot be seen by theories built on the metric field. We have in mind a vague analogy with gauge theories like QCD, descibed in terms of pions in the sigma-model or by quarks and gluons, respectively.

The unbroken phase of $2+1$ gravity is naturally described by the Chern-Simons theory [2]. This has a good ultra-violet behaviour, and the fundamental gauge fields $(e, \omega)$ fluctuate around vanishing v.e.vs.. The metric is not present in eq. (7), thus the theory is topological invariant.

However, in order to interpret this as a theory of gravity, one has to go backward in the steps eqs.(2-6) and define the metric by the soldering condition (2). This implies additional constraints on a naive treatment of the Chern-Simons theory. The condition (2) breaks topological invari-

\#4 Following Witten 12 . 
ance to reparametrization invariance, and it requires a classical (long-distance) limit of the theory dominated by smooth gauge configurations, yielding invertible metrics

$$
\left\langle\hat{e}_{\mu}^{a}\right\rangle \sim \frac{1}{G_{N}} \delta_{\mu}^{a}
$$

where $\hbar G_{N}$ is the minimal length for the breaking to take place. Notice that a dimensionful scale is not present in the (non observable) pure gauge theory (or pure gravity), but it is introduced by the mass of the particles, owing to eq. (4).

We believe that this is the main question, how the "good" Chern-Simons theory manages to reproduce geometry and the special role played by the Minkowski metric. Actually, in classical gravity the Minkowskian asymptotic behaviour is an additional physical condition not implied by the local Einstein equations. The natural guess is that this metric should emerge from the solution of the quantum theory.

As a side remark, let us show that the firstorder approach does not lead to a renormalizable theory in four dimensions, thus additional theoretical inputs are needed. We repeat the previous transformation on the Einstein action (1), using now the identity

$\epsilon^{\mu \nu \rho \sigma} \epsilon_{a b c d} e^{a}{ }_{\mu} e^{b}{ }_{\nu} e_{\rho}^{c} e^{d}{ }_{\sigma}=|e| \quad(D=4)$

and we obtain

$$
\begin{aligned}
S & = \\
& -\frac{1}{8} \int d^{4} x \epsilon^{\mu \nu \rho \sigma} \epsilon_{a b c d} e^{a}{ }_{\mu} e_{\nu}^{b}\left(\partial_{[\rho} \omega_{\sigma]}+\omega_{[\rho} \omega_{\sigma]}\right)^{c d} \\
& -2 \sum_{(r)} \int d \tau\left(\dot{\xi}^{\mu} P_{a} e^{a}{ }_{\mu}\right)_{(r)}
\end{aligned}
$$

The action is again polynomial, but it has no term quadratic in the fields. Therefore we cannot prove perturbative renormalizability, because we cannot expand around $e \sim \omega \sim 0$. The unbroken phase of gravity cannot be seen in the basic field variables, and one has to invoke more hypothetical models in order to keep this picture, like Topological Field Theories [12] and Pregeometric Models 13].

Another possibility is to set up a non-perturbative quantization method for the action (14). This is Ashtekar's approach 14. The proposal which attracted more attention is String Theory, which indeed reproduces gravity in the classical and high-energy limit [15].

Such additional theoretical structures are not needed in three-dimensional gravity, which has the virtue of simplicity. Physical problems common to four dimensions can be addressed directly, and hopefully solved.

\section{The Einstein-Infeld-Hoffmann Problem}

Let us recall the problem of motion for pointlike matter sources in gravity. The field equations of motion follow by varying the action (11) w.r.t. the metric

$G_{\mu \nu}=\mathcal{R}_{\mu \nu}-\frac{1}{2} \mathcal{R} g_{\mu \nu}=T_{\mu \nu}$

$T_{\mu \nu}=\sum_{(r)} \frac{p_{\mu}^{(r)} p_{\nu}^{(r)}}{m_{(r)}} \frac{\delta^{(2)}\left(\boldsymbol{x}-\boldsymbol{\xi}^{(r)}(t)\right)}{\sqrt{g}} \frac{d \tau}{d t}$

where the trajectories are parametrized by $\xi^{\mu}=$ $(t, \boldsymbol{\xi}(t))$, and the momenta are given in eq.(4).

In gauge theories like electrodynamics, the motion $\xi^{\mu}$ is subjected to the independent particle equation (the Lorentz force). In gravity instead, the particle (geodesic) equation is not independent, but follows from reparametrization invari- 
Fig. 1. (a) Minkowski space with excised region, and (b) its tail representation in the Chern-Simons solution. The trajectory of a test particle is also indicated.

ance of the field equations

$0=D_{\mu} T^{\mu \nu}$

$=\sum_{(r)}\left[\frac{d p^{\nu}}{d \tau}+\Gamma_{\alpha \beta}^{\nu}[\xi] p^{\alpha} p^{\beta}\right]_{(r)} \frac{\delta^{(2)}\left(\boldsymbol{x}-\boldsymbol{\xi}_{(r)}\right)}{m_{(r)} \sqrt{g}} \frac{d \tau}{d t}$

The difference is that in electrodynamics the particle equations are gauge invariant, while in gravity the geodesic equation (16) is reparametrization dependent \#5.

The Einstein equations (15) give the field as a functional of the particle motion $\left\{\xi_{(r)}\right\}$ and eq.116) gives the feedback, resulting in a strong non-linear problem. Moreover, the factor $\sqrt{g}$ implies that we cannot even write down the equations without a guess of the solution. This explains why this problem has only been solved perturbatively or for a single source.

In three dimensions it simplifies considerably, because the Ricci tensor determines uniquely the \#5 See e.g. chapters 65 and 106 of ref. 16.

\section{Riemann curvature}

$$
R_{\mu \nu, \rho \sigma}=-g \epsilon_{\mu \nu \lambda} \epsilon_{\rho \sigma \gamma} G^{\lambda \gamma}, \quad(D=3)(17)
$$

Therefore, the classical space-times are flat apart from singularities at the sources. For example [1], a static particle of mass $m$ produces the following metric, in coordinates $x^{\mu} \equiv(t, r, \varphi)$,

$d s^{2}=d t^{2}-d r^{2}-r^{2} \alpha^{2} d \varphi^{2} \quad, \quad 0 \leq \varphi<2 \pi,(18)$

This is a cone because the circumference-toradius ratio $2 \pi \alpha \equiv 2 \pi[1-(m / 2 \pi)]<2 \pi$, i.e. the deficit angle is equal to the mass. Alternatively, it can be represented as Minkowski space with an excised region

$d s^{2}=d T^{2}-d R^{2}-R^{2} d \phi^{2} \quad, \quad 0 \leq \phi<2 \pi \alpha,(19)$

where $\phi=\alpha \varphi, T=t, R=r$. The $X$-coordinates are singular, because the two edges of the excised region are indentified by a finite rotation (see Fig.1a)

$X_{+}^{\mu}=\left(e^{m \mathcal{J}_{0}}\right)^{\mu}{ }_{\nu} X_{-}^{\nu}$ 
which corresponds to a delta term in the metric, not written in eq.(19). Equation (20) is the wellknown matching condition of Deser, Jackiw and 't Hooft [1] \#6.

The metric of a particle with momentum $p$ can be obtained by performing a Lorentz boost $B(v)$. It is again Minkowskian, with generalized matching condition given by the Poincaré transformation

$$
\begin{aligned}
& X_{+}-\xi(t)=e^{p \cdot \mathcal{J}}\left(X_{-}-\xi(t)\right), \\
& \left(e^{p \cdot \mathcal{J}}=B(v) e^{m \mathcal{J}_{0}} B(v)^{-1}\right)
\end{aligned}
$$

For many particles, the flat space outside the sources can be taken again Minkowskian, with an excised region for each particle - neglecting for the moment their possible overlappings. This picture was put forward by the previous authors as a general solution of the motion. While simple and appealing, this solution is not acceptable because the metric is singular at the excised regions. Moreover, it leads to a puzzling free motion for two particles, because excised regions can never overlap.

\section{The Many-Anyon solution in Chern-Simons Theory}

As we said, singular metrics cannot be handled in the Einstein theory, but are natural in the gauge theory of $(e, \omega)$. The previous DeserJackiw-'t Hooft (DJH) picture of multi-excised Minkowski space better applies to the following solution of the anyon problem, in the simplest gauge. More general solutions with singular metric will be found in other gauges, which allow

\#6 Quantum mechanics and scattering on this space were discussed in refs. 17. for arbitrary motion of the particles. We shall also see what happens when two excised regions meet. In sect. 5, we shall discuss how to close the excised regions and obtain a smooth metric, namely how to undo the transformation from eq.(18) to eq.(19) in the many-particle case.

\subsection{Equations of Motion}

The Chern-Simons field equations are read off from the action (7)

$$
\begin{aligned}
R_{\mu \nu b}^{a} & \equiv\left(\partial_{[\mu} \omega_{\nu]}+\left[\omega_{\mu}, \omega_{\nu}\right]\right)_{b}^{a} \\
& =-\epsilon_{\mu \nu \lambda} \epsilon_{b c}^{a} \sum_{r} v_{r}^{\lambda} P_{(r)}^{c} \delta^{2}\left(x-\xi_{(r)}\right), \\
T_{\mu \nu}{ }^{a} & \equiv \partial_{[\mu} e_{\nu]}+\omega_{[\mu} e_{\nu]}=0
\end{aligned}
$$

These are the Cartan structure equations of the first-order formalism. The first one expresses the Riemann tensor in terms of the sources, as eq.(17), while eq.(23) gives a vanishing torsion \#7.

By introducing the $4 \times 4$ representation of the Poincaré group

$A_{\mu}=\left(\begin{array}{cc}\omega_{\mu b}^{a} & e_{\mu}^{a} \\ 0 & 0\end{array}\right), \mathcal{J}_{a}=\left(\begin{array}{cc}-\left(\epsilon_{a}\right)^{b} & 0 \\ 0 & 0\end{array}\right)$

one can easily see that the r.h.s. of eqs.(22,23) are the components of the (flat) connection $F_{\mu \nu}$ in the Chern-Simons theory.

First-order gravity and Poincaré gauge theory have the same gauge transformations, provided the equations of motion are used [2]. Therefore, the geodesic particle equation should follow similarly from local Poincaré invariance of the ChernSimons field equations. Actually, this implies the identity

\#7 For spinning particles, the torsion is proportional to the spin sources, thus the Chern-Simons theory corresponds to the more general Einstein-Cartan theory of gravity 5 . 
$\epsilon^{\mu \nu \rho} \mathcal{D}_{\rho} F_{\mu \nu}=0, \quad \mathcal{D}_{\rho} \equiv \partial_{\rho}+\left[A_{\rho}, \quad\right]$

which reads in components

$\dot{P}_{(r)}^{a}+\dot{\xi}_{(r)}^{\mu} \omega_{\mu b}^{a} P_{(r)}^{b}=0$

$\epsilon_{b c}^{a} P_{(r)}^{b} e^{c}{ }_{\mu} p_{(r)}^{\mu}=0$

The first component is recognized as the geodesic equation (16) in $(e, \omega)$ variables, by using eqs.(3. 5). The second one is similar to the kinematical relation (5), but less strong because it does not fix the mass of the particles. This breaks scale invariance and cannot follow from the ChernSimons theory, it should be added as an additional requirement, like the definition of the metric discussed before.

Notice that eqs. (26,27) are "charge" conservation equations in Chern-Simons, where the nonabelian charge is given by the Casimir $P^{2}=m^{2}$ (and $J \cdot P=m \sigma$ for non zero spins $\sigma_{(r)}$ ). They become dynamical equations due to the identification of gauge and space-time manifolds necessary for gravity. Therefore, we should not include the (non-abelian) Lorentz equation for the particles, as suggested by the analogy with the Yang-Mills theory, and done for anyons in condensed matter physics 18].

\subsection{The Solution}

To summarize, the Chern-Simons equations of motion are (5), (22), (23) and (26). The general solution will be characterized by constant Lorentz momenta $P_{(r)}^{a}=m_{(r)} U_{(r)}^{a}=$ const, and parallel (non-covariant) velocities $V_{(r)}^{i}=$ $U_{(r)}^{i} / U_{(r)}^{0}(i=1,2)$. The generalization to nonparallel ones and non-constant $P^{a}$ 's will be discussed later. The solution exists for arbitrary trajectories $\left\{x^{i}=\xi_{(r)}^{i}(t)\right\}$, which can be written

$$
X^{2}\left(\xi_{(r)}^{\mu}\right)=B_{(r)}=\text { const. }
$$$$
X^{1}\left(\xi_{(r)}^{\mu}\right)=V_{(r)} X^{0}\left(\xi_{(r)}^{\mu}\right)
$$

where $X^{a}\left(x^{\mu}\right)$ are arbitrary functions of the coordinates $x^{\mu}$, invertible at fixed time,

$J_{(r)}=\left|\frac{\partial\left(X^{1}-V_{(r)} X^{0}, X^{2}\right)}{\partial\left(x^{1}, x^{2}\right)}\right|_{(r)}>0$

Let us present the solution in the simplest gauge $X^{a}=\delta_{\mu}^{a} x^{\mu}$,

$\begin{aligned} \omega_{\mu} & =\sum_{(r)} \omega_{\mu}^{(r)} \\ e^{a}{ }_{\mu} & =\partial_{\mu} X^{a}+\sum_{(r)} \omega_{(r) \mu b}^{a}\left(X-B_{(r)}\right)^{b}\end{aligned}$

where, for each particle $(r)$,

$$
\begin{aligned}
\omega_{(r) \mu} & =\left(P_{(r)} \cdot \mathcal{J}\right)\left(\partial_{\mu} \Theta\left(X^{2}-B_{(r)}\right)\right) \\
& \times \Theta\left(V_{(r)} X^{0}-X^{1}\right)
\end{aligned}
$$

where $\Theta$ is the step-function. This solution is linear in the sources and satisfies the superposition principle. Any particle has associated a branch cut on the left, or "tail" (Fig.1b), the support of the spin connection. The tail location is a gauge choice and it can be freely rotated, provided another tail is not met.

Notice that $\left(1+\omega_{(r)}\right) \quad$ is an infinitesimal rotation around the constant vector $P_{(r)}$, therefore the term $\omega_{\mu} P$ in the geodesic equation (26) drops out, and our ansatz $P_{(r)}^{a}=$ const. is consistent. The field equations are easily verified, because the quadratic terms vanish for separated tails.

The solution for generic trajectories can be obtained by performing the arbitrary coordinate transformation $X^{a}\left(x^{\mu}\right)$. Actually, the equations of motion and the solution have an intrinsic expression in terms of differential forms, so that their relations are reparametrization invariant.

In the general case, the $P^{a}$ 's stay constant but the $p^{\mu}$ are not, thus the trajectories in spacetime bend and particles do interact. We clearly see that dynamics in three dimensions is not determined by the local equations of motion, but 
by the choice of gauge, subjected to more subtle global metric conditions.

Before coming to this point, let us show that our solution in the simplest gauge $X^{a}=\delta_{\mu}^{a} x^{\mu}$ reproduces Deser-Jackiw-'t Hooft excised spacetime. The geometry of such singular metric can be understood by studying the geodesics, i.e. by sending a beam of test particles. Their geodesic curves $x^{\mu}(\tau)$ also satisfy eq. (26), which can be integrated once

$e^{a}{ }_{\nu} \dot{x}^{\nu}(\tau)=P\left(\exp -\int_{\tau_{0}}^{\tau} d x^{\mu} \omega_{\mu}\right)_{b}^{a} U^{b}$

where $P$ is the path -ordering along the geodesic, and $U$ is a constant velocity. In the region outside tails $\omega_{\mu}=0$, thus the geodesics are straight lines. Across the tail, the tangent vector makes a jump. Near the r-th tail we can substitute eq.(31) in eq.(33), integrate again and obtain the matching condition (21), for a point just above $\left(X_{+}\right)$and just below $\left(X_{-}\right)$the tail (fig.1) 顿.

\subsection{Constants of Motion from the Holonomies}

The observables of the $\operatorname{ISO}(2,1)$ Chern- Simons theory are the loop integrals

$U_{\Gamma}(y, x)=P \exp \left(-\int_{\Gamma} \omega \cdot \mathcal{J}+e \cdot \mathcal{P}\right)$

where $\Gamma$ is an oriented path from $x$ to $y$. Gauge invariant quantities can be obtained from closed loops, by looking for quantities invariant under $U \rightarrow g U g^{-1}$. By parametrizing

$U_{\Gamma}(x, x)=\left(\begin{array}{cc}L=e^{-w \cdot \mathcal{J}} & q \\ 0 & 1\end{array}\right) \equiv(L, q, 1)$

we find the following two invariants

$$
\begin{aligned}
& \sqrt{w^{2}} \leftrightarrow \operatorname{Tr}_{(1)} L=1+2 \cos \left(\sqrt{w^{2}}\right) \\
& \sigma=\frac{q^{a} w_{a}}{\sqrt{w^{2}}}
\end{aligned}
$$

$\left(\sigma=q^{a} w_{a}\right.$ for $\left.w^{2}=0\right)$. The Lorentz invariant is the angle of the (pseudo)-rotation $\sqrt{w^{2}}$, the spin invariant is the projection of the translation $q^{a}$ on the rotation axis [9].

The loops are also invariant under smooth deformations of the metric, because they are given by the integral of a one-form. The particle motion being a kind of topological deformation, it will not affect their value. Thus the invariants (36, 37) are constants of motion of the particle plus field dynamics 119 .

Any particle $(r)$ has associated an elementary holonomy $U_{(r)}\left(x_{(r)}, x_{(r)}\right)$, for a tiny loop surrounding it counterclockwise, with basepoint $x_{(r)}$ near the particle, off the tail,

$$
\begin{aligned}
U_{(r)}\left(x_{(r)}, x_{(r)}\right) & =\exp \left(-\int_{\Sigma_{(r)}} d \sigma^{\mu \nu} F_{\mu \nu}\right) \\
& =\left(L_{(r)} \equiv e^{-\mathcal{J} \cdot P_{(r)}}, J_{(r)}, 1\right)(38)
\end{aligned}
$$

which can be computed from the field equations [4.5]. Its invariants (36, 37) are just the Casimirs of the particle $w^{2}=P_{(r)}^{2}=m_{(r)}^{2}$, and $\sigma=(P \cdot J / m)_{(r)}=\sigma_{(r)}$, independent of time as announced (hereafter $\sigma_{r}=0$ ).

Let us consider now two particles and the holonomy for the loop encircling them once counterclockwise. From the solution (30.31) we can compute its corresponding invariants, which are the rotation $\mathcal{M}$ and the spin $S$ invariants 晒,

$\cos \frac{\mathcal{M}}{2}=c_{(1)} c_{(2)}-s_{(1)} s_{(2)} \frac{P_{(1)} \cdot P_{(2)}}{m_{(1)} m_{(2)}}$

$S \sin \frac{\mathcal{M}}{2}=2 s_{(1)} s_{(2)} \epsilon_{a b c}\left(B_{(1)}-B_{(2)}\right)^{a} \frac{P_{(1)}^{b} P_{(2)}^{c}}{m_{(1)} m_{(2)}}$ where $c_{(i)}=\cos \frac{m_{(i)}}{2}, s_{(i)}=\sin \frac{m_{(i)}}{2}$.

These two formulas were obtained by Deser, Jackiw and 't Hooft [1]. Their recipe of composing matching conditions for each particle finds the correct meaning in the Poincaré holono- 
mies\#8. $\mathcal{M}$ and $S$ are two of the infinite conservation laws of the Chern-Simons theory.

In non-invariant terms, Deser, Jackiw and 't Hooft argued that there is a choice of coordinates, which at large space-like distances $|\underline{x}| \gg|\underline{B}|$ is Minkowskian with a single excised region and jump in time determined by $\mathcal{M}$ and $S$ in eq.(40), a kind of center-of-mass system. Actually, for $G_{N} m_{(r)} \ll 1, \mathcal{M}$ and $S$ reduce to the usual formulas of special relativity for the total invariant mass and the total angular momentum. Higher corrections were interpreted as global contributions of the gravitational field. However, this reasoning on singular metrics encounters a new difficulty, because inertial or accelerating center-of-mass systems should be distinguished by the asymptotic behaviour of the metric, which is undecidable in this case.

\subsection{Particle exchanges and the Yang- Baxter equation}

Let us now discuss how the solution extends to the case of non-parallel velocities, leading to crossings of tails. Without loss of generality, consider the case of particle (2) crossing the tail of particle (1) (fig.2). $P_{(2)}^{a}$ is no longer constant, and it evolves according to the integrated eq.(26)

$P_{(2)}\left(x^{+}\right)=P \exp \left(-\int_{x^{-}}^{x^{+}} \omega_{(1)}\right) P_{(2)}\left(x^{-}\right)$

where $x^{+}\left(x^{-}\right)$correspond to $t>0(t<0)$, the collision being at $t=0$. If we ignore the feedback of (2) on (1) (test-particle limit), this equation gives the matching condition of the geodesic eq. 21)

$P_{(1)}\left(x^{+}\right)=P_{(1)}\left(x^{-}\right)$

$P_{(2)}\left(x^{+}\right)=L_{(1)} P_{(2)}\left(x^{-}\right)$

\#8 See also ref. [8].
Fig. 2. The particle exchange operation $\sigma_{12}$, for the indicated orientation of tails.

Actually this result is also valid when both particles are dynamical 4 . It can be proven by using the integrated form of the field equations given by the non-Abelian Stokes theorem [18].

One should not think of the crossing of tails as the scattering of two particles. It might look so in this particular gauge $X^{a}=\delta_{\mu}^{a} x^{\mu}$, but it is possible to put it in an invariant form, which holds for all solutions in arbitrary gauge $X^{a}\left(x^{\mu}\right)$. Thus it involves only the topology of the trajectories and manifestly gives no conditions on the $p_{(r)}^{\mu}$ 's. Therefore, a correct name for the process of tail crossing is particle exchange.

More precisely, let us define a particle exchange operator $\sigma_{12}$ (fig.2) acting on the tensor space $V_{(1)} \otimes V_{(2)}$, which contains the elementary Poincaré holonomies of the two particles. They should have a common base-point, thus $U_{(2)}$ in eq. 38 is translated to $B_{(1)}$,

$U_{(1)}=\left(L_{(1)}, 0,1\right)$,

$U_{(2)}=\left(L_{(2)},\left(L_{(2)}-1\right)\left(B_{(1)}-B_{(2)}\right), 1\right)$

The effect of particle (2) crossing the tail of (1) can be written in the form 
Fig. 3. Sequence of exchanges for the Yang-Baxter equation.

$\sigma_{12}:\left\{\begin{array}{l}U_{(1)} \rightarrow U_{(1)} \\ U_{(2)} \rightarrow U_{(1)} U_{(2)} U_{(1)}^{-1}\end{array}\right.$

which is manifestly topological invariant. It is partially gauge dependent, because a different tail orientation, i.e. a different choice of basepoint, would have given the operator $\sigma_{21}$, thus exchanging the (asymmetric) role of the two particles. On the other hand, the monodromy of the particle (2) around (1), i.e. the double braiding $\sigma_{12} \sigma_{21}$, is gauge invariant $\# 9$.

Next, the exchanges of $N$ particles follow by composition of the operators $\sigma_{i, i+1}, i=1, \ldots, N-$ 1 , which generate the braid group $\mathcal{B}_{N}$. They act on tensor spaces $V_{(r)}$ of particle holonomies, all with the same basepoint. The generators $\sigma_{i, i+1}$ satisfy the Yang-Baxter equation

$\sigma_{i j} \sigma_{i k} \sigma_{j k}=\sigma_{j k} \sigma_{i k} \sigma_{i j}, \quad k=j+1=i+2$
$\sigma_{i, i+1} \sigma_{j, j+1}=\sigma_{j, j+1} \sigma_{i, i+1}, \quad|i-j| \geq 2$

where the first equation acts on $V_{(i)} \otimes V_{(j)} \otimes V_{(k)}$ and the second one on $V_{(i)} \otimes V_{(i+1)} \otimes V_{(k)} \otimes V_{(k+1)}$. This equation expresses associativity of particle

\#9 Similar investigations of the Braid group were done in the refs. [8], 18 . exchanges, as required by the deformability of trajectories (fig.3).

The Yang-Baxter equation is not trivial in our non-Abelian case, and it provides a consistency check of our previous analysis on crossings [4. Clearly, it should be an identity at the classical level, because conditions on the momenta would mean a classical motion only for some initial conditions.

At the quantum level, one should find a representation of the Braid Group in the Hilbert space, and show that the physical states are in the trivial singlet representation. This strong condition has not yet been solved in the nonAbelian case 20,21.

\section{Smooth metrics and the $\Lambda$-transformation}

Let us now look at the gravitational problem, for which the behaviour of the metric $g=e^{T} \eta e$ is important. We want to avoid the $\delta$-function 
singularities present in the general solution 30 32 ), i.e. to close the excised regions discussed before.

\subsection{One Particle}

In the case of a static particle, the angular rescaling from (18) to (19) can be interpreted as the transformation

$X=\exp \left(\frac{m}{2 \pi} \varphi(x) \mathcal{J}_{0}\right) x=\Lambda(x) x$

The $\Lambda$ rotation varies from $-m / 2$ to $m / 2$ when $\varphi$ varies from $-\pi$ to $\pi$ and thus generates a solution to the matching condition for the $X$-variable in eq. 20). As a consequence, $x$ is continuous, as it should be. These continuous coordinates $x^{\mu}$ correspond to a choice of gauge for $X(x)$ in the general solution (31), which gives the dreibein

$$
\begin{aligned}
e_{\mu}^{a} & =\left[\left(\partial_{\mu}+\omega_{\mu}\right) \Lambda x\right]^{a}= \\
& =\Lambda_{b}^{a}{ }_{b}\left(\delta_{\mu}^{b}+\frac{m}{2 \pi} n^{b} n_{\mu}\right),
\end{aligned}
$$

where $n_{\mu}=\left(0, \epsilon_{i j} \hat{x}^{j}\right)$. After some computations, the metric becomes the one in eq.(18), as expected.

For the moving particle, the $\Lambda$-transformation is obtained by applying a Lorentz boost to the static transformation (45) 化. A particularly interesting limit is the massless case, with fixed energy E, obtained by letting

$m \rightarrow 0 \quad(\gamma \rightarrow \infty)$, with $m \gamma=E$

The result is the Aichelburg-Sexl metric [22] for a massless particle in three dimensions,

$d s^{2}=2 d u d v-(d y)^{2}+\sqrt{2} E \delta(u)|y|(d u)^{2}$

where the light-cone variables are

$u=\frac{t-x}{\sqrt{2}}, \quad v=\frac{t+x}{\sqrt{2}}$
Notice that the Lorentz contraction has transformed the smooth angular dependence in eq. (45) into a step-function,

$\Lambda \rightarrow \begin{cases}e^{-p \cdot \mathcal{J} / 2} & -\pi<\varphi<-\pi / 2 \\ 1 & -\pi / 2<\varphi<\pi / 2 \\ e^{p \cdot \mathcal{J} / 2} & \pi / 2<\varphi<\pi\end{cases}$

producing a metric (48) with a (now physical) singularity at the wave-front, or shock wave (fig.4).

\subsection{Geodesic Scattering}

The $\Lambda$-mapping is particularly useful to describe the motion of a test particle in the oneparticle metric described before. In fact we already know that, in $X$-coordinates, the geodesics are straight lines, if they do not cross the tail.

If $\phi$ denotes the azimuthal $X$-coordinate in the rest frame, eq.(19), we obtain by inverting eq. 45.,

$x^{a}(\tau)=\Lambda^{-1}\left(\frac{\phi(\tau)}{\alpha}\right)_{b}^{a}\left(U^{b} \tau+B^{b}\right)$

It is thus easy to discuss the asymptotic motion and scattering of the test particle. The asymptotic velocity becomes

$$
\begin{aligned}
u_{ \pm}(\infty) & =\Lambda^{-1}\left( \pm \frac{\pi}{\alpha}\right) u_{ \pm}(-\infty) \\
& =e^{ \pm p \cdot \mathcal{J} / 2 \alpha} u_{ \pm}(-\infty)
\end{aligned}
$$

where the $+(-)$ sign holds according to whether the geodesic runs above (below) the field particle. Special cases of eq.(52) are:

i) The geodesic scattering angle off a particle of mass $m$ at rest [1]

$\theta_{0}=\frac{m}{2 \alpha}=\frac{m}{2}\left(1-\frac{m}{2 \pi}\right)^{-1}$.

iii) The scattering angle for both massless particles 
$\tan \frac{\theta}{2}=\frac{E}{2}$

where $E$ is the energy of the field-particle, in agreement with the derivation from the Aichelburg-Sexl metric 22].

The above results show that the gravitational problem for test particles is characterized by quantities which are not purely topological, and in our case are given in terms of the Christoffel connection by

$\operatorname{Pexp}\left(-\int_{-\infty}^{\infty} d x_{\mu} \Gamma_{ \pm}^{\mu}\right)_{ \pm}=[L(P)]^{ \pm \frac{1}{2 \alpha}}$

where the path runs along the geodesic above (below) the point source of momentum $P$.

One may wonder to what extent this result is gauge dependent.

In principle, one may choose to change the $\Lambda$-transformation in the particle rest frame, by some reparametrization of the azimuthal variable $\varphi$. This, however, will make the scattering angle in general dependent on the direction of the probe in an arbitrary way. Since this violates the physical notion of isotropy of space for a spinless particle, we shall explicitly exclude it in the following.

In other words, we require on physical grounds the $\Lambda$-transformation to be given (at large distance, i.e., for $r \rightarrow \infty$ ) by eqs.(45), which define the isotropic single-particle metric in our framework. Then the result (55), due to the properties of parallel transport, will be invariant under any local reparametrization which is asymptotically consistent with our choice.

To summarize, in the singular $X$-coordinates the geodesic scattering is ambiguous, because of the possibility that a branch-cut is crossed. On the other hand, in the smooth $x$-coordinate, the geodesic scattering angle is uniquely determined in the rest-frame (with the isotropic choice) and therefore also in any other frame, giving rise to the more general "S-matrix" in eq.(55).

\subsection{Two-Particle Scattering}

Let us now consider the problem of finding the gauge fixing $X^{a}=\bar{X}^{a}\left(x^{\mu}\right)$ which gives a smooth metric $g=e^{T} \eta e$ in the case of two dynamical particles. This is more difficult than the previous one-particle transformation (45), and we do not yet have a complete solution. Nevertheless, we shall state the gauge conditions which, in our opinion, are sufficient to obtain a non-ambiguous result for the scattering process. These conditions admit a solution in the first perturbative order in $G m_{(r)}$. Under the assumption that an exact solution exists, we shall give an ansatz for the exact scattering angle in the massless case[4].

These gauge conditions are as follows:

- Smoothness conditions.

The gauge fixing is supposed to be of the form

$\bar{X}^{A}=(\mathcal{T}(x))_{B}^{A} x^{B}, \quad(A=0,1,2,3)$

where $\mathcal{T}(x)$ is a Poincaré transformation which builds a solution of the matching conditions for each particle,

$\mathcal{T} \simeq \mathcal{T}_{(r)}(x) S_{(r)}(x), \quad x^{\mu} \simeq \xi_{(r)}^{\mu}(\tau)$,

where

$\mathcal{T}_{(r)}=e^{B_{(r)} \cdot \mathcal{P}} \Lambda_{(r)}\left(\varphi_{(r)}\right) e^{-B_{(r)} \cdot \mathcal{P}} \quad(r=1,2)$
$\Lambda_{(r)}=\exp \left(\frac{\varphi_{(r)}(x)}{2 \pi} P_{(r)} \cdot \mathcal{J}\right)$

is the (properly translated) single particle transformation discussed in the previous section, and $S_{(r)}$ is instead regular at that point. From the definition (58) it follows that, close to $\xi_{(r)}^{\mu}, \mathcal{T}$ performs the change of variables which closes the excised region, as in eq.(45). In the case of scattering $P_{(1)} \neq P_{(2)}$, the non-commutativity of the $\Lambda_{(r)}$ 's is the main difficulty in finding the exact form of $\mathcal{T}$. 
- Asymptotic conditions.

Further conditions correspond to the definition of asymptotic states. The commutator of $\Lambda_{(1)}$ and $\Lambda_{(2)}$ becomes vanishingly small when the particles are far apart, thus we can impose the initial condition

$\mathcal{T}(x) \rightarrow \mathcal{T}_{(1)}(x) \mathcal{T}_{(2)}(x) \simeq \mathcal{T}_{(2)}(x) \mathcal{T}_{(1)}(x)$

for fixed spatial coordinates and large negative times. This ensures that the class of metrics we are interested in is asymptotically consistent with the gauge fixing for a single particle given in the previous section.

By inspection, the momenta of the incident particles are the constants $P_{(1)}, P_{(2)}$ of our solution,

$p_{(1)}^{\mu}(-\infty)=\delta_{a}^{\mu} P_{(1)}^{a}, \quad p_{(2)}^{\mu}(-\infty)=\delta_{a}^{\mu} P_{(2)}^{a}$,

Moreover, one can check that the parallel transport from (1) and (2) to the central region $|x| \ll T$ is trivial. Therefore, in this region we can define, as in special relativity, the total momentum,

$p^{\mu}=p_{(1)}^{\mu}+p_{(2)}^{\mu}$,

$p_{\mu}=g_{\mu \nu}(-\infty) p^{\nu}=\eta_{\mu \nu} p^{\nu}$

the invariant mass squared $s=\left(p_{(1)}+p_{(2)}\right)^{2}$, and finally the center-of-mass frame by

$\left(p_{(1)}+p_{(2)}\right)^{i}=0, \quad i=1,2$.

An additional asymptotic condition is needed, at fixed time and large $|x|$, in order to avoid rotating frames at infinity. This requires that the Christoffel connection $\Gamma_{\mu}$ is as small as dimensionally allowed,

$\Gamma_{\mu}=O\left(\frac{1}{|\underline{x}|}\right) \quad(|\underline{x}| \rightarrow \infty, T$ fixed $)$.

In contrast, in a rotating frame $\Gamma_{\mu}$ would be of order $\left(\Omega^{2}|\underline{x}|\right)$.

These gauge fixing conditions admit a nontrivial solution in perturbation theory, $G E_{(r)} \ll 1$.
This is analogous to the "fast", weak coupling, approximation used in four dimensions [6]. The Lorentz part $\Lambda$ of the transformation $\mathcal{T}$ in eq.(56) is approximately of product type

$\Lambda \simeq \Lambda_{(1)} \Lambda_{(2)} \simeq \Lambda_{(2)} \Lambda_{(1)}$

because the commutator terms are of 2 nd order in the $G E_{(r)}$ 's. This takes care of both the polydromy and initial state conditions to this order. Finally there are no rotations at infinity, because the angles $\varphi_{(r)}$ are body-fixed with the straightmoving particles, thus eq. 62) is verified.

The first-order center-of-mass scattering angle turns out to be [A]

$\theta=\frac{\sqrt{s}}{2}\left(1+O\left(G m_{(r)}\right)\right), \quad\left(8 \pi G_{N}=1\right)$

in agreement with the classical limit of string scattering [15.

The zero mass, or high energy limit $m_{(r)} \ll \sqrt{s}$, has also some simplifying features. The $\Lambda$-transformation has the form of a shock wave, eq.(50), with no effect on times earlier than the arrival of the particle. Thus we can superpose two oneparticle Aichelburg-Sexl metrics with Minkowski space in-between, both before and after the collision of wave-fronts at $T=0$. This is shown in fig.4, where the $\Lambda$ is constant in each sector and takes the values $L_{r}^{ \pm 1 / 2}=\exp \left(\mp p_{(r)} \cdot \mathcal{J}\right)$, with $p_{(1)}=(E, E, 0), p_{(2)}=(E,-E, 0)$.

Since the $P_{(r)}$ 's are constant, and therefore the discontinuities across the wavefronts and tails are fixed, the $t>0 \Lambda$-mapping is still piecewise constant in the regions bounded by wavefronts and tails (50), and uniquely determined by the polydromy requirements (58) up to the following Lorentz transformation

$R^{-1}(\theta)=\left.\lim _{\epsilon \rightarrow 0} P \exp \left(-\int_{t=-\epsilon}^{t=+\epsilon} \Gamma_{\mu} d x^{\mu}\right)\right|_{\underline{x}=0}$ 
Fig. 4. (a) Initial and (b) final state pattern of the $\Lambda$-transformation for the scattering of two massless particles. (The tail and wave-front singularities of $\Lambda$ are indicated by curly and straight lines).

which gives the classical S-matrix in this case. Notice that the $X$-coordinates stay constant at $T=0$, because the two tails do not cross.

$R^{-1}$ is determinated by the coordinate condition that there are no rotations at infinity at $T=0$ in the $x$-coordinates external to the wavefronts 活. We obtain the rotation angle

$$
\tan \frac{\theta}{2}=\frac{E}{2}=\frac{\sqrt{s}}{4} \quad\left(8 \pi G_{N}=1\right) .
$$

Notice that this angle is the same as the geodesic one, eq.(54), but the corresponding S-matrices (55,65) are quite different, in particular the latter one is in agreement with energy conservation.

\section{Conclusions and Outlook}

We have shown that the first-order approach to three-dimensional gravity, rephrased in the Chern-Simons gauge theory, allows a larger class of solutions of the classical field theory, because reparametrization invariance need not be completely fixed.

This has clearly shown that the trajectories of particles are gauge degrees of freedom. They are arbitrary, apart from the topological constraint given by the Braid group \#10. Therefore the classical motion in gravity is completely determined by the gauge choice.

There are no natural gauge choices in the classical Chern-Simons theory. Similarly, the unbroken phase seems to be the natural one in the quantum theory.

On the other hand, we have enphasized that many aspects of the classical motion in the Einstein theory are gauge dependent, and that there are physical gauges, for which the metric is smooth, isotropic, and asymptotically inertial. For gravitational scattering, it is sufficient to choose the physical gauge asymptoti-

\#10 And the condition of eq.(29). 
cally (asymptotic breaking), because the scattering angle can be written as the holonomy for an open time-like path, eqs. (55) and (65), which is invariant under localized reparametrizations.

Therefore, from the classical point of view, the unbroken phase of gravity at short distance and the broken one at large distance are not incompatible, provided one understands the quantum mechanism for this asymptotic breaking.

This can only arise from the coupling of quantized matter to gravity, because the pure gravity case does not show it [2]. The known actions of matter coupled to gravity are based on the equivalence principle, and need an invertible metric field, therefore they cannot be used in the unbroken phase. An $I S O(2,1)$-invariant coupling of matter to first-order gravity is not yet known. This problem can possibly be by-passed by quantizing the degrees of freedom of the point sources, in the effective action obtained by our general classical solution. In this kind of Coulomb gas approach, the main problem to start with is the invariance of Hilbert space under the Braid group, as mentioned before 20,21.

\section{Acknowledgements}

I would like to thank my collaborators in this work, Marcello Ciafaloni and Paolo Valtancoli, and Daniele Amati for interesting discussions. I also thank the Organizers of this Workshop and the Departament ECM, University of Barcelona, for their kind hospitality.

\section{References}

[1] S. Deser, R. Jackiw and G. 't Hooft, Ann. Phys. (NY) 152 (1984) 220.

[2] E. Witten, Nucl. Phys. B311 (1988-89) 46, B323 (1989) 113.
[3] J. R. Gott, III, Phys. Rev. Lett. 66 (1991) 1126; S. Deser, R. Jackiw and G. 't Hooft, Physical Cosmic Strings do not generate Closed Time-Like Curves, preprint MIT-CPT-2011, to appear in Phys. Rev. Lett.

[4] A. Cappelli, M.Ciafaloni and P.Valtancoli, Classical Scattering in $2+1$ Gravity with $N$ Point Sources, preprint CERN-TH-6093/91, to appear in Nucl. Phys. B.

[5] A. Cappelli, M.Ciafaloni and P.Valtancoli, Classical Scattering in $2+1$ Gravity with $N$ Spinning Sources, preprint CERN-TH-6249/91;

[6] A. Einstein, L. Infeld and B. Hoffmann, Ann. Math. 39 (1938) 65;

J.Goldberg, in Gravitation, Introduction to current research, p.102, L.Witten editor, Wiley, London 1962.

[7] R. Jackiw, Five Lectures on Planar Gravity, preprint MIT-CPT-1936; Update on Planar Gravity (Physics of Infinite Cosmic Strings), preprint MIT-CPT-1986.

[8] S. Carlip, Nucl. Phys. B324 (1989) 106.

[9] P. Sousa Gerbert, Nucl. Phys. B346 (1990) 440.

[10] K.Koehler, F.Mansouri, C.Vaz and L.Witten, Nucl. Phys. B348 (1991) 373.

[11] G.Grignani and G.Nardelli, Gravity in $2+1$ Dimensions Coupled to Point-like Sources: A flat Chern-Simons Gauge Theory equivalent to Einstein, preprint MIT CPT-1953 (1991).

[12] E. Witten, Com. Math. Phys. 117 (1988) 353.

[13] D. Amati and G. Veneziano, Nucl. Phys. B204 (1982) 451.

[14] A.Ashtekar, New Perspectives in Canonical Gravity. Lecture Notes (Bibliopolis, Napoli 1988);

C. Rovelli, Ashtekar formulation of general relativity and loop-space non-perturbative quantum gravity: a report, to appear in Phys. Reports.

[15] D. Amati, M. Ciafaloni and G.Veneziano, Int. J. Mod. Phys. 3A (1988) 1615; Nucl. Phys. B347 (1990) 550.

[16] L.D.Landau and E.M.Lifsits, The Classical Theory of Fields, (Pergamon, Oxford, 1962).

[17] G. 't Hooft, Comm. Math. Phys. 117 (1988) 685; S. Deser and R. Jackiw, Comm. Math. Phys. 118 (1988) 495;

P. Sousa Gerbert and R. Jackiw, Comm. Math. Phys. 124 (1989) 229. 
[18] A.P. Balachandran,M. Bourdeau and S. Jo, Int. J. Mod. Phys. A5 (1990) 2423;

K. Gupta and A. Stern, Spin and statistics in $2+1$ gravity, preprint Syracuse, SU-4228-463 (1991).

[19] V. Moncrief, J. Math. Phys. 30 (1989) 2907.

[20] E. Witten, Comm. Math. Phys. 121 (1989) 351;

A. Schwimmer, in Recent developments of CFT, ICTP Trieste October 89, S.Randjbar-Daemi et al. eds., World Scientific, Singapore 1990.

[21] E. Verlinde, A note on braid statistics and the nonAbelian Aharonov-Bohm effect, preprint IASSNSHEP-90/60;

E. Guadagnini and F.Volpi, Two-Particle Scattering Amplitude in Gauge ISO(2,1) Quantum Gravity, preprint Pisa IFUP-TH-29/91.

[22] T. Dray and G. 't Hooft, Nucl. Phys. B253 (1985) 173 ;

V. Ferrari, P. Pendenza and G. Veneziano, Gen. Rel. Grav. 20 (1988) 1185. 